\title{
Article \\ Structural Diversity, Fitness Cost, and Stability of a Bla NDM-1-Bearing Cointegrate Plasmid in Klebsiella pneumoniae and Escherichia coli
}

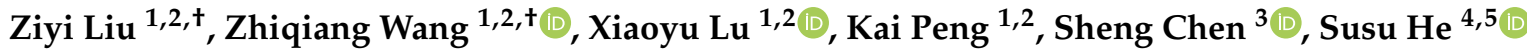 \\ and Ruichao $\mathrm{Li}^{1,2, *(D)}$
}

1 Jiangsu Co-Innovation Center for Prevention and Control of Important Animal Infectious Diseases and Zoonoses, College of Veterinary Medicine, Yangzhou University, Yangzhou 225009, China; lzy519461865@yeah.net (Z.L.); zqwang@yzu.edu.cn (Z.W.); Luxiaoyu2017happy@163.com (X.L.); pkai94@sina.com (K.P.)

2 Institute of Comparative Medicine, Yangzhou University, Yangzhou 225009, China

3 Department of Infectious Diseases and Public Health, Jockey Club College of Veterinary Medicine and Life Sciences, City University of Hong Kong, Hong Kong 999077, China; shechen@cityu.edu.hk

4 State Key Laboratory of Pharmaceutical Biotechnology, Medical School of Nanjing University, Nanjing 210093, China; susuhetian@nju.edu.cn

5 Jiangsu Laboratory of Molecular Medicine, Medical School of Nanjing University, Nanjing 210093, China

* Correspondence: rchl88@yzu.edu.cn

+ These authors contributed equally to this work.

check for updates

Citation: Liu, Z.; Wang, Z.; Lu, X.; Peng, K.; Chen, S.; He, S.; Li, R. Structural Diversity, Fitness Cost, and Stability of a $B l a_{\mathrm{NDM}-1}$-Bearing Cointegrate Plasmid in Klebsiella pneumoniae and Escherichia coli. Microorganisms 2021, 9, 2435. https://doi.org/10.3390/ microorganisms 9122435

Academic Editor: Michael F. Hynes

Received: 28 October 2021

Accepted: 23 November 2021

Published: 25 November 2021

Publisher's Note: MDPI stays neutral with regard to jurisdictional claims in published maps and institutional affiliations.

Copyright: (c) 2021 by the authors. Licensee MDPI, Basel, Switzerland. This article is an open access article distributed under the terms and conditions of the Creative Commons Attribution (CC BY) license (https:/ / creativecommons.org/licenses/by/ $4.0 /)$.

\begin{abstract}
Cointegrate/hybrid plasmids combine the genetic elements of two or more plasmids and generally carry abundant antimicrobial resistance determinants. Hence, the spread of cointegrate plasmids will accelerate the transmission of AMR genes. To evaluate the transmission risk caused by cointegrate plasmids, we investigated the structural diversity, fitness cost, and stability of a cointegrate plasmid in Klebsiella pneumoniae YZ6 and Escherichia coli EC600. The cointegrate plasmid pSL131_IncA/C_IncX3 was from a clinical Salmonella Lomita strain. After transferring the plasmid into E. coli EC600 by conjugation, we observed plasmids with different structures, including a full-length original plasmid and two truncated versions. By contrast, DNA fragment deletion and bla $_{\mathrm{CTX}-\mathrm{M}-14}$ gene insertion in the plasmid were detected in a transconjugant derived from K. pneumoniae YZ6. These results suggest that the structure of the plasmid was unstable during conjugation. Furthermore, both the full-length plasmid in EC600 and the structurally reorganized plasmid in YZ6 imposed a fitness cost on the bacterial host and enhanced biofilm formation ability. Serial passaging in antibiotic-free medium resulted in a rapid decline of the plasmid in YZ6. However, the stability of the structurally reorganized plasmid in YZ6 was improved via serial passaging in antibiotic-containing medium. SNP calling revealed that mutations of the outer membrane porin may play an essential role in this process. These findings indicate that structural versatility could contribute to the dissemination of cointegrate plasmids. Although the plasmid incurred a fitness cost in other Enterobacteriaceae species, positive selection could alleviate the adverse effects.
\end{abstract}

Keywords: $b l a_{\mathrm{NDM}-1}$; cointegrate plasmid; fitness cost; structural diversity; plasmid stability

\section{Introduction}

Plasmids, which are important drivers of bacterial evolution, code a wide range of traits that assist hosts in better adapting to complicated niches and stresses [1]. The function of plasmids in the dissemination of antimicrobial resistance (AMR) genes, facilitating the acquisition of multiple resistance genes by pathogens in a single-transfer event, is of great importance in various settings [2]. Generally, horizontally obtained AMR genes or multidrug-resistant (MDR) plasmids can impose an obvious fitness cost on the host [3], and are expected to be unstable during bacterial growth. Comparatively, plasmids can persist 
stably in bacterial populations in the absence of selection for plasmid-encoded traits, which is known as the plasmid paradox [4]. In laboratory settings, while plasmid persistence is usually observed, the level of selection required by plasmid persistence varies from no selection to different levels of antibiotic selection [5]. For example, bla $a_{\mathrm{CTX}-\mathrm{M}-14 \text {-carrying }}$ plasmid pCT, could persist and disseminate in the absence of antibiotic pressure [6]. Nontransmissible plasmid pNUK73, initially found in P. aeruginosa PAO1, was unstable, but compensatory adaptation increased its stability by alleviating the cost of plasmid carriage under a period of selection [1]. In addition, plasmid pKP33 was markedly less stable in naïve hosts (clinical strains that did not carry multidrug resistance plasmids) compared to the original host, but plasmid-host co-adaptation occurred in the presence of antibiotic selection, which improved its persistence after removal of selective pressure [7].

While the adaptation of small, non-conjugative plasmids is usually improved by selection pressure and compensatory adaptation, most AMR plasmids carry their own stability systems, such as toxin-antitoxin and partitioning systems, which confer stability in the bacteria by post-segregational killing or growth inhibition. Generally, the classical antitoxin forms a complex with the toxin to block its effects, and the TA complex is passed on to daughter cells. If the daughter cells do not inherit the plasmid, they are no longer able to produce more toxin or antitoxin molecules. With the degradation of unstable antitoxins, the toxins will exert their cytotoxic or growth inhibitory effects to ensure the maintenance of the plasmid in the cell population [8,9]. In addition, several hypotheses have been proposed to resolve plasmid persistence, such as host-plasmid co-adaptation, plasmid hitchhiking, cross-ecotype transfer, and high plasmid transfer rates; however, none of them could adequately clarify the plasmid paradox [5].

Plasmid fusion is increasingly detected in clinical and environmental bacterial isolates. Mobile elements are the main contributors to plasmid replicon fusion. Bacterial insertion sequences applying replicative transposition fuse transposon-bearing and target plasmids as a cointegrate [10]. In addition, mobile elements presenting as multiple copies can act as hot spots for homologous recombination. For instance, the presence of IS26 in two plasmids of a carbapenem-resistant NDM-5-producing E. coli isolate led to plasmid fusion via homologous recombination [11]. Unlike traditional plasmids, due to the combination of genetic information of original plasmids, fusion/hybrid plasmids possess more AMR genes and insertion sequences with high plasticity. In recent years, the emergence of fusion/hybrid plasmids has been frequently reported, including tet (X4)-bearing plasmids [12], mcr-1-bearing plasmids [13,14], and other AMR genes carrying plasmids [15], causing serious public concern, as these plasmids play a crucial role in the dissemination of AMR genes. In this study, we investigated the structural diversity of a $b l a_{\mathrm{NDM}-1}$-bearing cointegrate plasmid during conjugation and assessed the fitness cost and stability in order to systematically evaluate the transmission potential of this plasmid (Figure 1). 


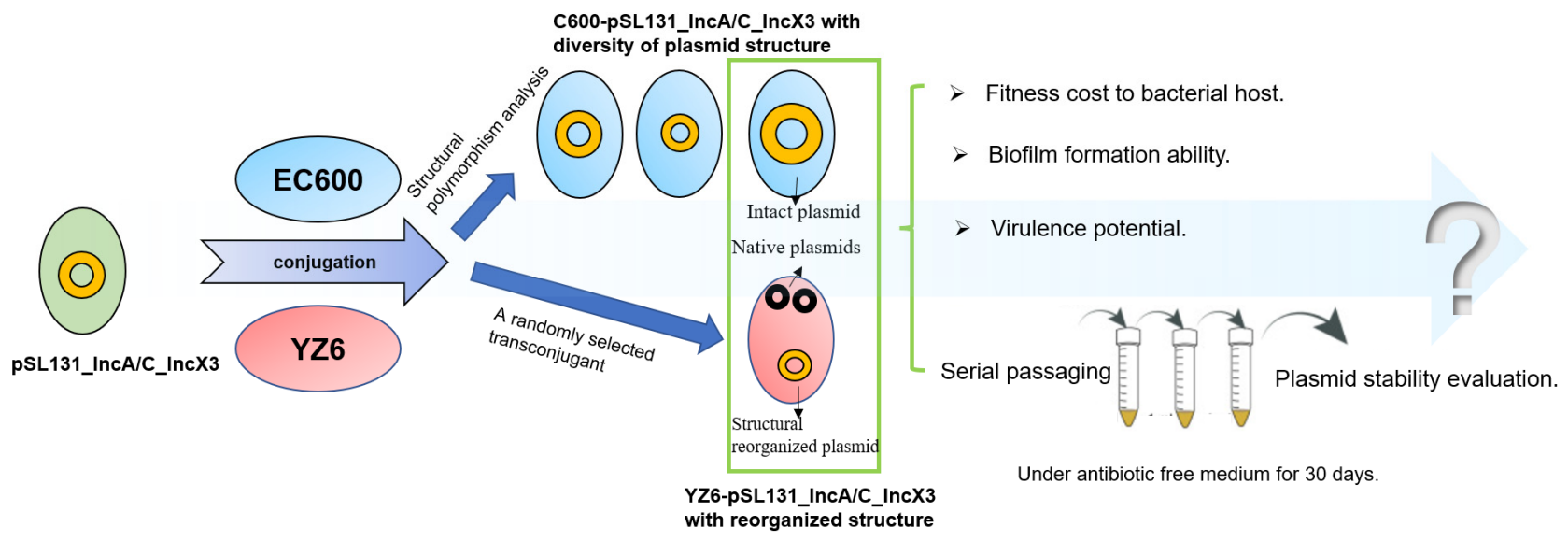

Figure 1. Schematic diagram of research process. A cointegrate plasmid (pSL131_IncA/C_IncX3) was transferred by conjugation into Escherichia coli EC600 and Klebsiella pneumoniae YZ6. Analysis of structural polymorphism was conducted after plasmid acquired by EC600. EC600 carrying intact plasmid and YZ6 carrying structural reorganized plasmid were subjected to fitness evaluation and serial passaging under antibiotic-free conditions for 30 days. Yellow circles in different sizes represented changes in cointegrate plasmid size during conjugation.

\section{Materials and Methods}

\subsection{Bacterial Strains and Plasmids}

The basic information of the cointegrate plasmid pSL131_IncA/C_IncX3 with different structures, including the presence of TA or partitioning systems, in this study is listed in Table S1. Strain YZ6 was a derivative of classical ST11 carbapenem-resistant K. pneumoniae HS11286 (accession number for the HS11286 chromosome is CP003200, and the six plasmids are CP003223 to CP003228) collected from sputum samples [16], and their characteristics are listed in Table S2. Salmonella Lomita SL131 was the natural host of the cointegrate plasmid pSL131_IncA/C_IncX3 [17], and rifampin-resistant E. coli EC600 was used as the recipient strain [18].

\subsection{Filter Mating Assay and S1-PFGE}

To investigate the structural diversity of pSL131_IncA/C_IncX3, conjugation assay was carried out between SL131 and EC600 using a filter mating method [19]. Transconjugants were selected on two types of antibiotics containing plates, one supplemented with meropenem and rifampicin (MR) and one supplemented with meropenem, tetracycline, and rifampicin (MTR). To characterize the profiles of cointegrate plasmids, SL131 and the corresponding transconjugants were digested with S1 nuclease (Takara, Osaka, Japan), followed by PFGE with the CHEF Mapper XA system (Bio-Rad, Hercules, CA, USA). Salmonella Braenderup H9812 standard strain restricted with XbaI was used as the molecular marker [20]. Conjugation assay was also performed between SL131 and YZ6. Transconjugants were screened by plates supplemented with hygromycin $(200 \mathrm{mg} / \mathrm{L})$, meropenem $(2 \mathrm{mg} / \mathrm{L})$, and tetracycline $(16 \mathrm{mg} / \mathrm{L})$.

\subsection{Growth Curve Measurements}

Overnight cultures of plasmid-free and plasmid-carrying strains were diluted to an optical density at $600 \mathrm{~nm}(\mathrm{OD} 600)$ of 0.05 , and the diluents were grown at $37^{\circ} \mathrm{C}$ for $12 \mathrm{~h}$ with vigorous aeration $(200 \mathrm{rpm})$. The culture cell density was determined every hour and measured by a Thermo Scientific Multiskan FC Microplate photometer at OD600. All experiments were repeated 3 times.

\subsection{Pairwise Competition Assay}

Overnight cultures of plasmid-bearing strains and their plasmid-free isogenic ancestors were diluted to $0.5 \mathrm{McF}$ arland standard and mixed at a ratio of 1:1 in $5 \mathrm{~mL}$ LB broth 
(Haibo Biotechnology Co., Ltd., Qingdao, China) [21]. Then, the mixtures were incubated at $37^{\circ} \mathrm{C}$ for $72 \mathrm{~h}$ with shaking. Every $24 \mathrm{~h}, 5-\mu \mathrm{L}$ cultures were reinoculated in $5 \mathrm{~mL}$ of fresh LB medium. The number of cells for each strain was determined by spreading serial 10-fold dilution onto LB agar plates with or without $2 \mathrm{mg} / \mathrm{L}$ of meropenem and $16 \mathrm{mg} / \mathrm{L}$ of tetracycline, and the relative fitness was calculated as follows: $w=\ln (\mathrm{NRt} / \mathrm{NR} 0) / \ln (\mathrm{NSt} / \mathrm{NS} 0)$, where NR is the number of resistant clones and NS is the number of susceptible clones, with values below 1 indicating the fitness cost.

\subsection{Galleria Mellonella Larval Infection Assay}

For Galleria mellonella larval infection assay, about $300 \mathrm{mg}$ of larvae was stored in a special box at $4{ }^{\circ} \mathrm{C}$ until use. Overnight cultures were washed and adjusted to $10^{6} \mathrm{CFU} / \mathrm{mL}$ using PBS. Ten larvae in each group were challenged with $10 \mu \mathrm{L}$ of diluent, and PBS was used as the negative control. Infected larvae were incubated in sterilized Petri dishes at $37^{\circ} \mathrm{C}$ for $72 \mathrm{~h}$, and the survival rate was recorded every $24 \mathrm{~h}$.

\subsection{Biofilm Formation}

Biofilm formation assay was conducted as previously described [22]. Overnight cultures were adjusted to a cell density equivalent to a $0.5 \mathrm{McFarland}$ standard. Then $200 \mu \mathrm{L}$ of each culture was transferred to a 96-well plate in triplicate. After being incubated at $37^{\circ} \mathrm{C}$ for 2 days, the cultures were discarded. Each well was washed twice with $200 \mu \mathrm{L}$ of PBS. The biofilms were fixed in methanol for $10 \mathrm{~min}$. Subsequently, each well was stained with $1 \%$ crystal violet solution for $10 \mathrm{~min}$ and rinsed with PBS until colorless. Finally, biofilms were dissolved in $100 \mu \mathrm{L}$ of $30 \%$ formic acid for $30 \mathrm{~min}$, and biofilm formation was quantified by measuring the absorbance at OD590.

\subsection{Plasmid Stability Experiments}

First, the cointegrate plasmid-bearing EC600 and YZ6 were propagated by serial passaging for 30 days in antibiotic-free LB broth. Every 12 h, $5 \mu \mathrm{L}$ of each culture was transferred to $5 \mathrm{~mL}$ of fresh LB broth. To evaluate the stability of the cointegrate plasmid, the fraction of plasmid-containing cells in population was calculated every 5 days by counting the number of colonies that grew on antibiotic-free and antibiotic-containing plates.

Subsequently, the cointegrate plasmid-carrying YZ6 strain was passaged for 30 days under meropenem and tetracycline pressure. The plasmid persistence was determined for 8 days as previously described with minor modification [23].

\subsection{DNA Sequencing and Bioinformatics Analysis}

The genomic DNA of the ancestral and three evolved strains was extracted using the TIANamp bacterial DNA kit (TianGen, Beijing, China) and subjected to short-read sequencing $(2 \times 150 \mathrm{bp})$ with the Illumina HiSeq 2500 platform. Short-read Illumina raw sequences of ancestral and evolved strains were separately assembled using SPAdes [24], and contigs less than 500 bp were discarded. SNP analysis was performed using Snippy (4.0.2) against the genome of the ancestral strain [25].

The plasmids of the three transconjugants of EC600, as well as pSL131_IncA/C_IncX3133K and pSL131_IncA/C_IncX3-ev1, were extracted using a Qiagen Plasmid Midi-Kit (Qiagen, Hilden, Germany). Subsequently, the plasmids were sequenced with the Oxford Nanopore Technologies MinION long-read platform. The nanopore long-read MinION sequences of plasmids were subjected to de novo assembly with the Flye tool [26]. Among them, raw sequences of pSL131_IncA/C_IncX3-133kb less than $30 \mathrm{~kb}$ were discarded before the assembly. BRIG and Easyfig were used to describe the structural diversity of cointegrate plasmid during conjugation $[27,28]$. 


\subsection{Statistics}

GraphPad Prism (6.0.1) was used to compare growth curves, relative fitness, biofilm formation ability, survival rate, and plasmid stability. Differences in survival were compared using the log-rank (Mantel-Cox) test. Absorbance values for biofilm formation between strains with and without the cointegrate plasmid were compared using the $t$-test. Statistical significance was set at $p<0.05$.

\section{Results and Discussion}

\subsection{Structural Diversity of Cointegrate Plasmid pSL131_IncA/C_IncX3 in EC600} after Conjugation

Plasmid pSL131_IncA/C_IncX3 of Salmonella Lomita origin about $216 \mathrm{~kb}$ in size was a bla $a_{\mathrm{NDM}-1}$-bearing cointegrate plasmid consisting of IncX3 and IncC plasmid backbones [17].

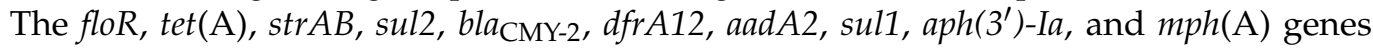
were located on IncC plasmid backbone, while $b l a_{\mathrm{NDM}-1}$ was detected on IncX3 plasmid backbone. In addition, it contained a type II TA system relE-Xre-like and partition module parA/parB, which are known to stabilize plasmid after replication and partitioning [29]. Our previous study revealed that the structure of the plasmid was prone to resolve single IncX3 plasmid during conjugation [17], yet the dynamic changes of the structure under the selection of other antibiotic combinations remained to be investigated.

To probe the structural polymorphism of the plasmid during conjugation, a conjugation assay was performed using EC600 Rif ${ }^{\mathrm{R}}$ as recipient strain. We randomly selected 11 transconjugants from MTR plates and 30 transconjugants from MR plates. Consistent with the previous study [17], a plasmid about $53 \mathrm{~kb}$ in size was detected in the 30 transconjugants from MR plates, suggesting that only the resolved IncX3 plasmid was present in them (Figure S1). By contrast, pSL131_IncA/C_IncX3 exhibited various sizes in the 11 transconjugants from MTR plates, which might be the consequence of plasmid reorganization (Figure S2). In general, some transconjugants from MR plates should be of MTR phenotype. However, the transconjugants from the two types of plates showed distinct differences. This was largely due to the selection-free IncC plasmid backbone triggering the resolution of fusion plasmid, and IncC plasmid was immediately discarded by the recipient strain during conjugation. To explore the underlying molecular mechanism of plasmid reorganization, three representative plasmids in transconjugants from MTR plates with different sizes were selected for nanopore sequencing.

Plasmid pMDRG14 shared a similar genetic structure with the original plasmid, indicating that the plasmid could transfer into the recipient strain with a full-length version (Figure 2a). Compared to the original plasmid, pMDRG21-157K discarded an approximately $59 \mathrm{~kb}$ region, including $a p h(3)-I a, m p h(\mathrm{~A})$, heavy metal tolerance mer operon, Rel-Xre-like TA system, and IncC replicon. Thus, the plasmid belonged to IncX3 type, rather than the original multireplicon type. This event was mediated by IS26, which plays a critical role in the dissemination of antibiotic resistance genes and formation of complex antibiotic resistance regions in Gram-negative bacteria [30]. The last plasmid, pMDRG11$112 \mathrm{~K}$, deleted a $105 \mathrm{~kb}$ region, including the aforementioned region, as well as a $39 \mathrm{~kb}$ region containing an integron between DNA recombination gene bet and IS26, and a $7 \mathrm{~kb}$ region containing partitioning gene parA located between IS26 and a hypothetical protein. The deletion leading to the plasmid formation was flanked by short sequence homologies (9 bp) on two genes with unknown function (Figure $2 b$ ). These findings indicate that the structure of pSL131_IncA/C_IncX3 was highly plastic and could be enriched under different antibiotic selection during conjugation. Conjugative events play an important role in the development of large plasmids, promoting plasmid evolution [31]. Considering that a limited number of transconjugants were selected and characterized, the possibility of other types of plasmid reorganizations could not be fully investigated. Nevertheless, our results at least highlight the high-plasticity trait of the plasmid, which may be regarded as the driving force in plasmid transmission and evolution. 
(a)

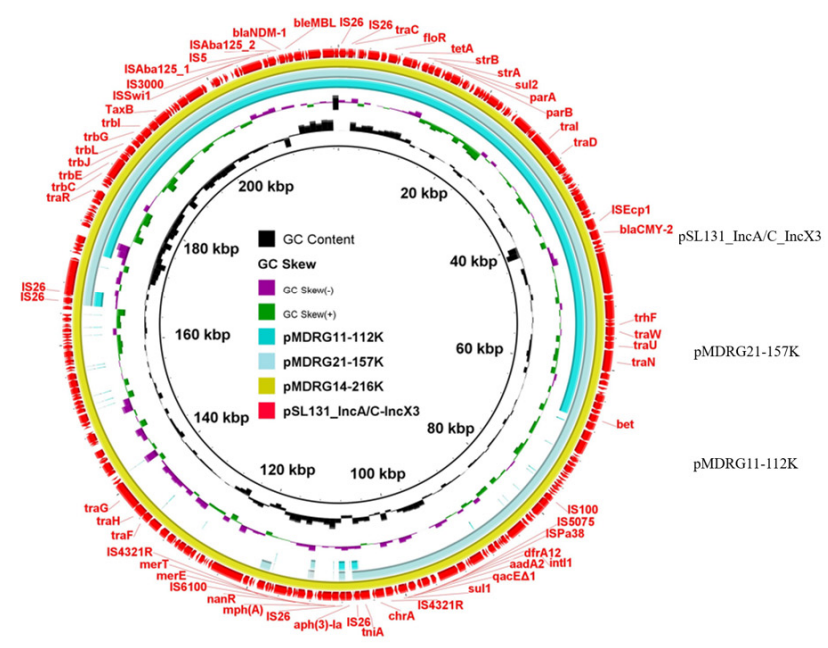

(b)

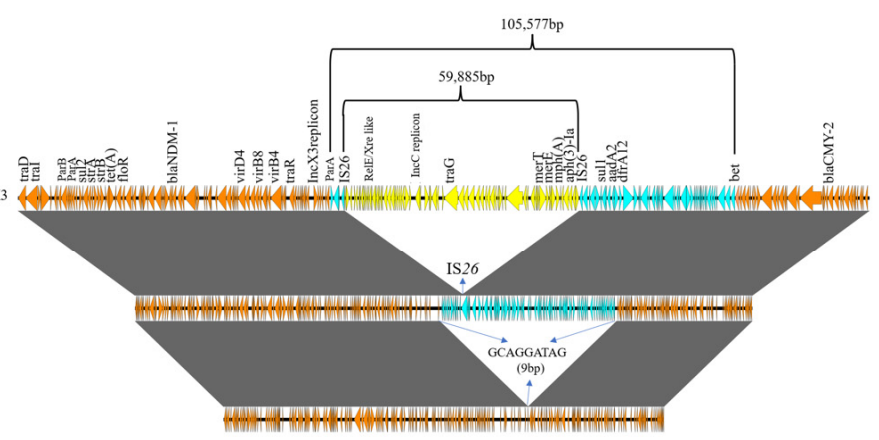

Figure 2. (a) Circular comparison of cointegrate plasmid pSL131_IncA/C_IncX3 with three plasmids from transconjugants of EC600 selected by MTR plates. Outermost circle with red arrows represents reference pSL131_IncA/C_IncX3. (b) Linear comparison of cointegrate plasmid pSL131_IncA/C_IncX3 with two structurally altered plasmids. Arrows marked in yellow indicate discarded regions from pSL131_IncA/C_IncX3 to pMDRG21-157K, and regions marked in yellow and blue represent discarded regions from pSL131_IncA/C_IncX3 to pMDRG11-112K.

\subsection{Cointegrate Plasmid pSL131_IncA/C_IncX3 Experienced Structural Reorganization in K. pneumoniae YZ6 after Conjugation}

To investigate the fitness cost of this cointegrate plasmid imposed on other Enterobacteriaceae hosts, the plasmid was further conjugated into K. pneumoniae $\mathrm{YZ6} \mathrm{Hyg}^{\mathrm{R}}$. A transconjugant named YZ6-pSL131_IncA/C_IncX3-133K was randomly selected for Nanopore MinION long-read sequencing. It carried the cointegrate plasmid with $133,188 \mathrm{bp}$, in which two regions were discarded in the IncC plasmid backbone. One of the regions was $66 \mathrm{~kb}$ and consisted of various genes encoding plasmid conjugative transfer protein, $b l a_{\mathrm{CMY}-2}$ gene, mobile elements, and hypothetical protein. The region was replaced by a gene that encoded the IS3 family transposase from the chromosome of YZ6. The other region contained $d f r A 12$, aadA2, sul1, aph(3)-Ia, and $m p h(\mathrm{~A})$ genes and heavy metal tolerance mer operon, and it was flanked by $7 \mathrm{bp}$ short sequence homologies. Moreover, the sequence of gene-encoding RHS repeat protein was partially lost, resulting in gene truncation. This deficiency was different from that in EC600, suggesting that the high variability of the plasmid structure may help the plasmid adapt to different host bacteria.

In addition, the plasmid acquired a Tn1721-derived structure carrying $b l a_{\mathrm{CTX}-\mathrm{M}-14}$ from native plasmid pKPHS1 of YZ6 [16]. The Tn1721-derived structure inserted into gene encoding DNA replication terminus site-binding protein and generated a $10 \mathrm{bp}$ direct repeat as the target site duplication upon mobile element insertion (Figure 3). After genetic recombination, the plasmid updated the profiles of AMR genes and insertion sequences, which raised concern about the emergence of novel plasmid. A recent study revealed that bla $_{\text {CTX-M-27 }}$ was able to transfer through transposition of a Tn1721-like structure between plasmids of Salmonella and E. coli [32]. Moreover, our findings prove that the transposon could transfer between different plasmids within bacteria. Tn1721-like transposons were found in numerous Gram-negative bacterial genomic or plasmid sequences [33]. Apart from $b a_{\mathrm{CTX}} \mathrm{M}, \mathrm{Tn} 1721$-like structure was also found to be associated with the dissemination of $f \circ s A 3, b l a_{\mathrm{KPC}-2}$, and tetracycline resistance genes [34,35]. These findings indicate the wide distribution of the transposon among clinical settings, and it is necessary to continuously monitor the plasmid evolution events mediated by this transposon. 


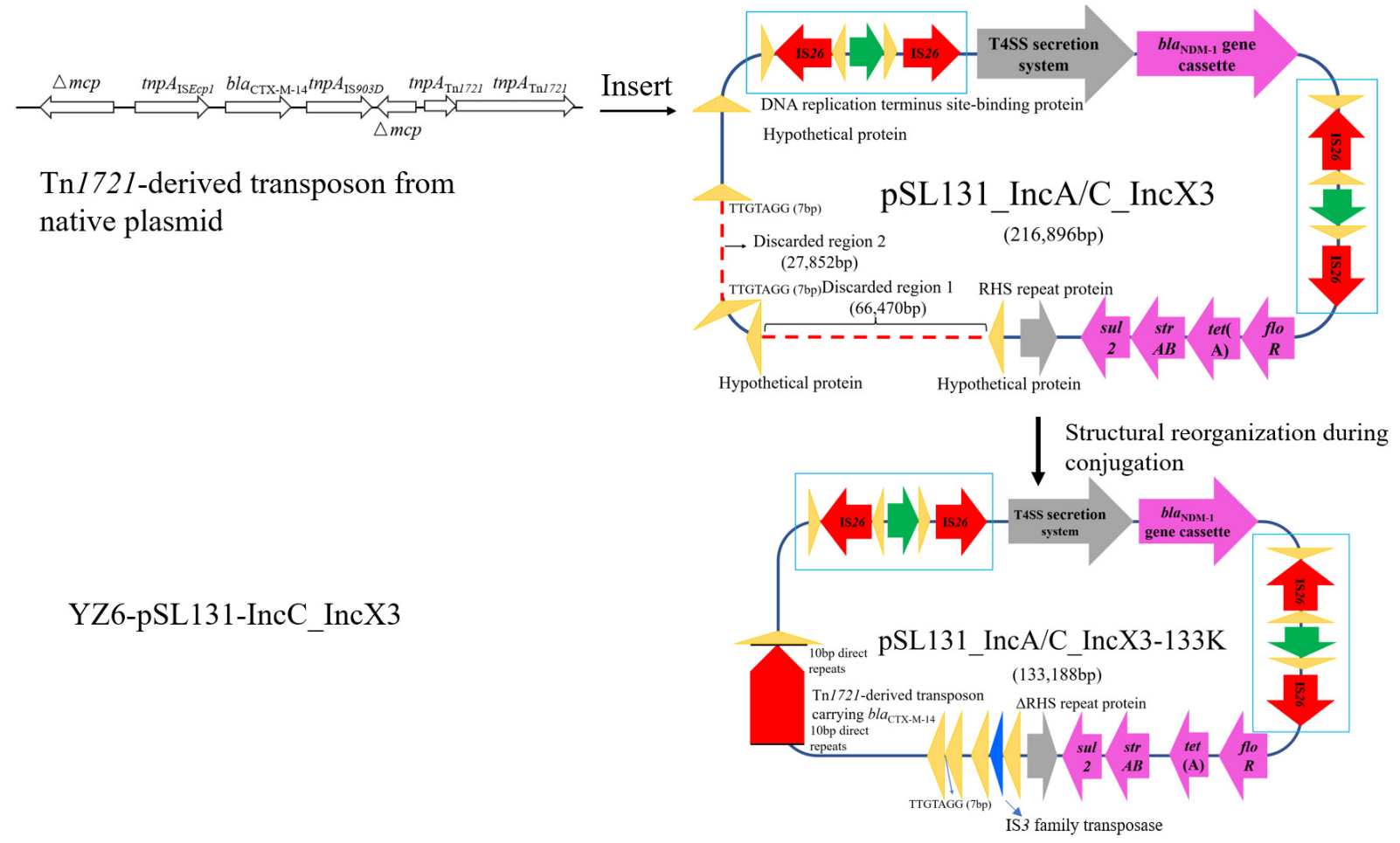

Figure 3. Schematic diagram of structural reorganization of pSL131_IncA/C_IncX3 after being conjugated into YZ6. Regions marked with dashed lines indicated discarded sequences; $66 \mathrm{~kb}$ was replaced by gene encoding IS3 family transposase. Deletion of the $27 \mathrm{~kb}$ region was mediated by short sequence homologies. The bla $\mathrm{CTX}_{\mathrm{M}-\mathrm{M}-14}$ gene from native plasmid was inserted into a gene-encoding DNA replication terminus site-binding protein mediated by Tn1721-derived transposon and left two $10 \mathrm{bp}$ direct repeats.

3.3. Cointegrate Plasmid pSL131_IncA/C_IncX3 Could Impose Different Levels of Fitness Cost in Klebsiella pneumoniae and E. coli

The transconjugant YZ6-pSL131_IncA/C_IncX3-133K and aforementioned MDRG14 (EC600-pSL131_IncA/C_IncX3) were subjected to fitness cost evaluation. According to the bacterial growth curves, we observed that the plasmid caused an obvious decrease in growth rate of EC600 and YZ6 (Figure 4a,b). Pairwise competitions were conducted between plasmid-bearing strains and their plasmid-free isogenic ancestors. The relative fitness of both YZ6 and EC600 was less than 1, suggesting that the plasmid imposed a fitness cost on EC600 and YZ6 (Figure 4c). Several studies have reported the fitness effects after acquisition of MDR plasmid on host bacteria. A bla $a_{\mathrm{KPC}-2}$ carrying cointegrate plasmid pT18 was found to affect the growth condition of E. coli DH5 $\alpha$ [36]. On the contrary, significantly increased fitness in E. coli $\mathrm{DH} 5 \alpha$ was observed after co-introduction of two plasmids harboring $b l a_{\mathrm{NDM}-1}$ and bla $a_{\mathrm{OXA}-232}$ [37]. Our data suggest that the plasmid imposed an extra burden on both YZ6 and EC600, which partly hindered the prevalence of the plasmid.

In addition, we compared the virulence and biofilm formation ability between the strains with and without the cointegrate plasmid. YZ6-pSL131_IncA/C_IncX3-133K showed decreased virulence compared to YZ6 $(p<0.01)$, which could be because the reduced fitness is expected to impair the bacterial virulence [38]. Nevertheless, the introduction of cointegrate plasmid did not affect the virulence of EC600 (n.s.). Biofilm formation assay revealed that both YZ6 and EC600 had significantly increased biofilm formation ability after plasmid acquisition $(p<0.5)$ (Figure 5). Biofilms play an important role in bacterial infection and the spread of AMR genes [39], especially in clinical settings, where they contribute to the expansion of various pathogens and AMR genes [40]. For instance, there was already evidence that the formation of biofilm in Staphylococcus aureus could promote the horizontal transfer of plasmid-borne AMR genes [41]. Thus, if the 
(a)

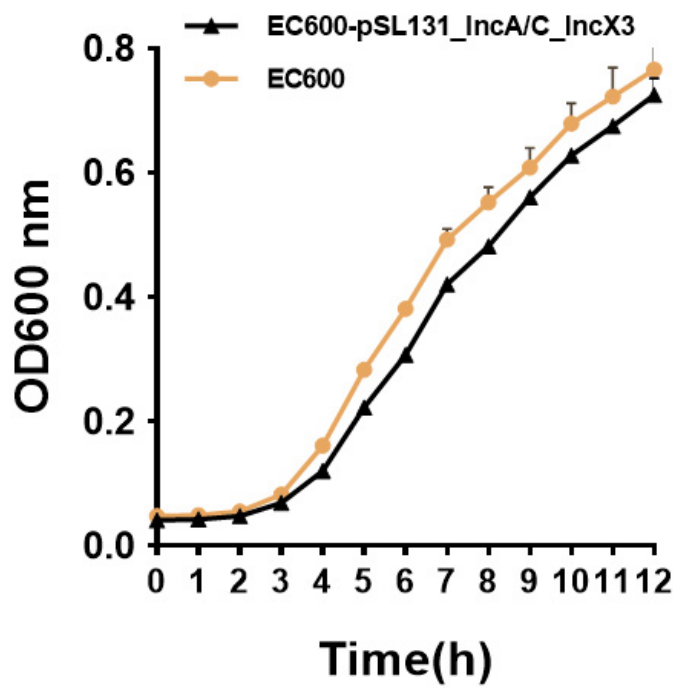

(c)

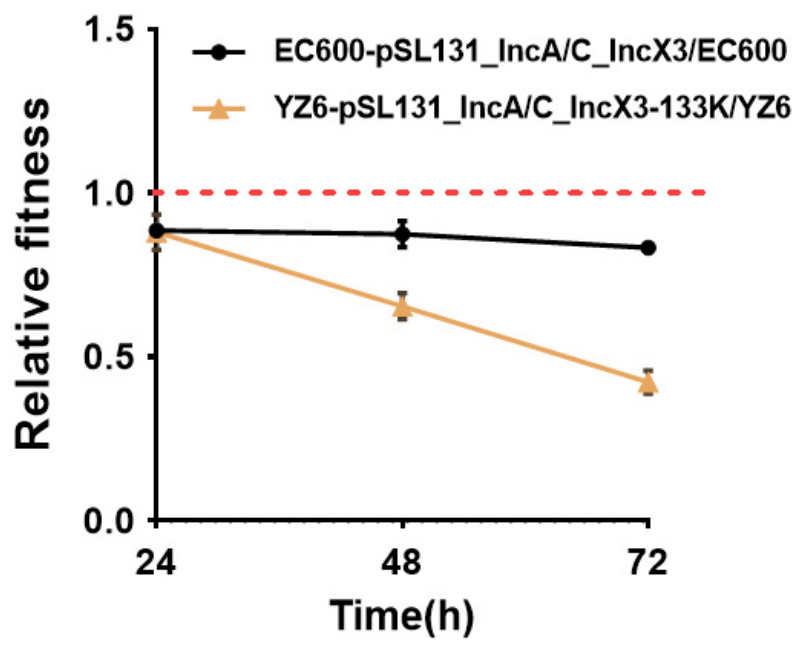

plasmid were widely distributed among clinical settings, it would inevitably complicate treatment and prevention measures.
Time(h)

(d)

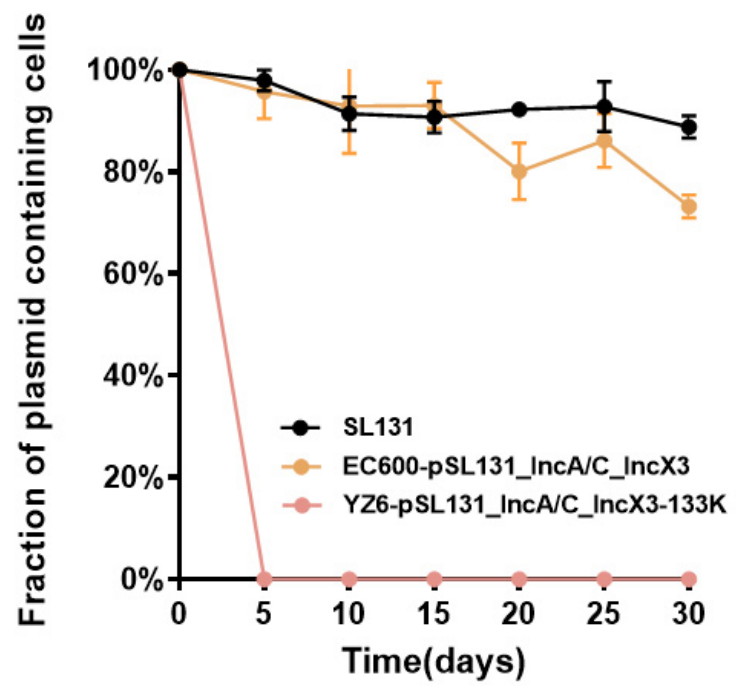

Figure 4. Initial fitness cost after acquisition of cointegrate plasmid in EC600 and YZ6 strains and plasmid persistence results of SL131, EC600-pSL131_IncA/C_IncX3, and YZ6-pSL131_IncA/C_IncX3-133K. (a,b) Growth curves of EC600 and EC600-pSL131_IncA/C_IncX3, YZ6 and YZ6-pSL131-IncA/C-IncX3-133K. (c) Relative fitness of two pSL131_IncA/C_IncX3 carrying strains. Relative fitness value less than 1 indicates fitness defect, and a value greater than 1 indicates fitness benefit. (d) Plasmid stability in bacterial population after serial passaging in antibiotic-free medium. Each point is the mean of three individual replicates, and error bars show standard deviation.

\subsection{Stability of Plasmids in YZ6, EC600, and Natural Host in Antibiotic-Free Medium}

In order to evaluate the stability of pSL131_IncA/C_IncX3 in its natural host SL131, as well as in YZ6 and EC600, in the absence of antibiotics, we propagated the plasmidharboring SL131, YZ6, and EC600 in antibiotic-free LB broth for 30 days. The plasmid could be stably maintained in its natural host and EC600 in at least 70\% of cells. The persistence of the plasmid in the natural host was largely a consequence of the long-term co-evolution of host and plasmid [17]. However, the plasmid was extremely unstable in YZ6, since rapid decline of the plasmid was observed within 5 days (Figure $4 \mathrm{~d}$ ). Both partitioning systems and TA systems were observed in the reorganized plasmid pSL131_IncA/C_IncX3133K (Table S1). However, they were unable to counteract the fitness cost imposed by 
plasmid in the host, resulting in plasmid loss during bacterial cell division. Furthermore, a recent study revealed that plasmid loss was determined by transcription-replication conflicts, and abiotic factors, such as temperature, should also be considered as factors influencing plasmid persistence [42], since the transcription-replication conflicts frequently arose following the acquisition of new genes (i.e., the insertion of mobile genetic elements could disrupt plasmid replication and thus lead to plasmid instability and extinction), and cold temperature appeared to be favorable for plasmid persistence.

(a)

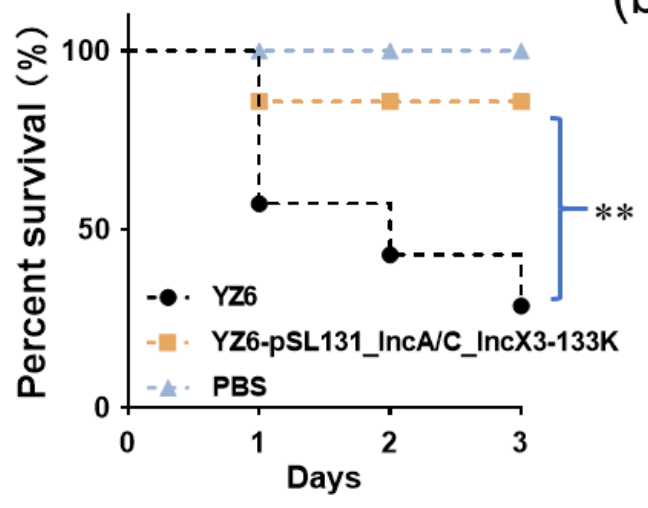

(b)

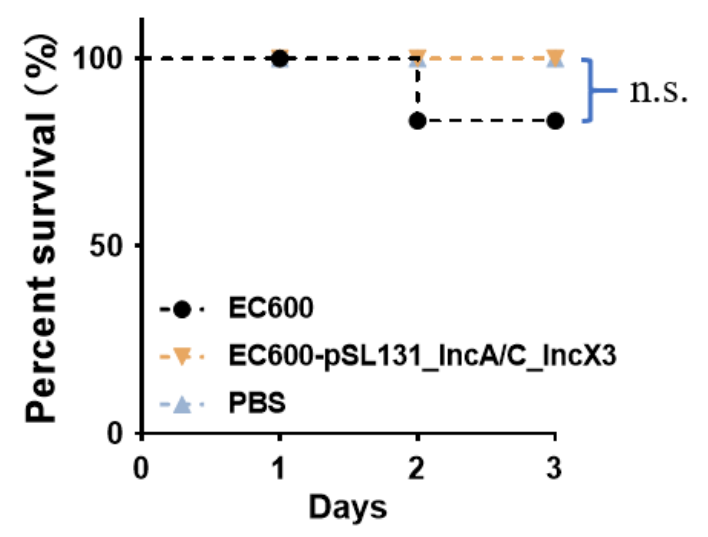

(c)

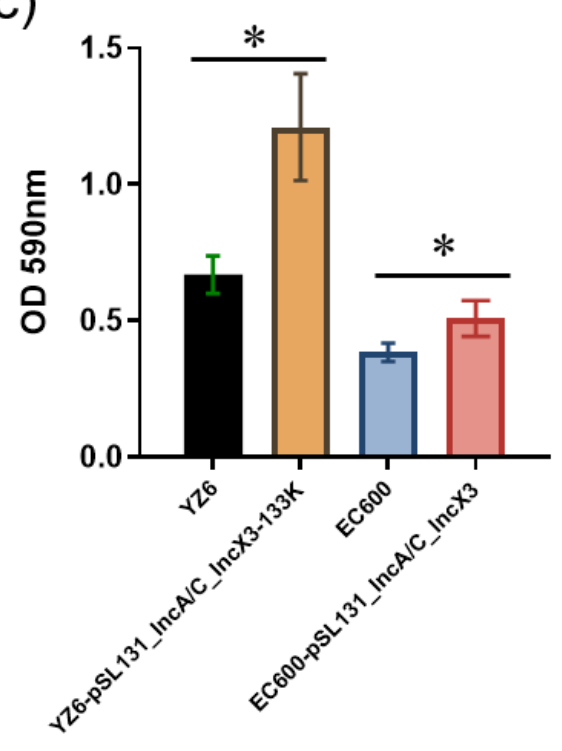

Figure 5. Results of virulence assay and biofilm formation ability of $Y Z 6$ and EC600, and their corresponding transconjugants. (a) Virulence potential of YZ6 and YZ6-pSL131_IncA/C_IncX3-133K in a G. mellonella infection model. ${ }^{* *} p<0.01$. (b) Virulence potential of EC600 and EC600-pSL131_IncA/C_IncX3 in a G. mellonella infection model. n.s, no difference. (c) Biofilm formation ability of YZ6 and YZ6-pSL131_IncA/C_IncX3-133K, EC600 and EC600-pSL131_IncA/C_IncX3. ${ }^{*} p<0.05$.

\subsection{Stability of Structural Deficiency Plasmid Was Improved in YZ6 under Positive Selection}

Given that pSL131_IncA/C_IncX3-133K was unlikely to persist in K. pneumoniae YZ6 in antibiotic-free medium, we performed a serial passaging experiment in LB broth containing meropenem $(2 \mathrm{mg} / \mathrm{L})$ and tetracycline $(16 \mathrm{mg} / \mathrm{L})$ for 30 days. After evolution under positive selection, three randomly selected clones were subjected to persistence assay in antibiotic-free LB broth for 8 consecutive days. While the plasmid was initially unstable in host bacteria, it was improved after long-term evolution, as the plasmid-containing 
cells dominated the cells by more than $70 \%$ after eight days of antibiotic-free passaging (Figure S3).

To investigate the potential mechanisms for the improved plasmid persistence, we performed whole-genome sequencing and analyzed the SNPs between ancestral and evolved strains. SNPs were located on chromosomes, and no SNPs were found in plasmids (Table S3). The chromosomal mutations of all evolved strains occurred in genes encoding hypothetical protein and outer membrane porin (OMP), and the SNP number of OMPs in YZ6-pSL131_IncA/C_IncX3-ev2 and YZ6-pSL131_IncA/C_IncX3-ev3 was higher than that in YZ6-pSL131_IncA/C_IncX3-ev1. Besides, no additional rearrangements were detected in evolved plasmid (Figure S4).

Large conjugative plasmids have three evolutionary patterns to increase persistence: mutations to replication genes, mutations to the global transcriptional regulatory system of the host, and changes in the plasmid backbone (i.e., deletions of costly regions of plasmid and acquisition of a toxin-antitoxin transposon) [5]. Several studies have demonstrated that imposing positive selection on plasmids usually resulted in compensatory evolution via plasmid or host chromosome mutations that facilitated the plasmid's persistence [43-45]. As previously mentioned, OMPs were strongly associated with antibiotic resistance, and the degree of expression was also related to the fitness cost of the bacteria [46]. Moreover, singlepoint mutation alone was sufficient to affect OMP function [47]. Thus, we hypothesized that the OMP mutations were likely to ameliorate the fitness cost of the host during evolution and promote plasmid survival.

Although this study highlights the importance of a cointegrate plasmid in AMR transmission, it still has some limitations. First, the structural diversity of the plasmid under different antibiotic pressures needs to be further explored. Second, the host species used in this study are limited. Third, the underlying mechanism leading to improved plasmid stability under positive selection still warrants further investigation.

\section{Conclusions}

Overall, this study revealed that the cointegrate plasmid pSL131_IncA/C_IncX3, with high plasticity, could flexibly discard various regions and acquire other AMR genes in response to a complex environment, demonstrating its strong dissemination and evolution potential. Transferring this plasmid to clinic-associated strains could increase the biofilm formation ability, which would inevitably increase the treatment failure rates. The imposition of fitness cost could limit the spread of the cointegrate plasmid. However, the plasmid could stably maintain under high-risk pathogenic strain after exposure to antibiotic pressure, which emphasizes that antibiotic residue is an indispensable driving force in the development of MDR pathogens.

Supplementary Materials: The following are available online at https:/ /www.mdpi.com/article/10 .3390 / microorganisms9122435/s1, Figure S1: S1-PFGE of transconjugants from MR plates. Figure S2: S1-PFGE of transconjugants from MTR plates. Figure S3: Persistence of pSL131_IncA/C_IncX3$133 \mathrm{~K}$ in three evolved clones after serial passaging in antibiotic-free medium. Figure S4: Circular comparison of cointegrate plasmid pSL131_IncA/C_IncX3 with two plasmid pSL131_IncA/C_IncX3133K and pSL131_IncA/C_IncX3-ev1. Table S1: Basic information of pSL131_IncA/C_IncX3 with different structures. Table S2: Basic information of HS11286 and YZ6. Table S3: SNPs identified in three evolved strains as compared with the ancestral strain.

Author Contributions: Z.W. and R.L. designed the experiments. Z.L. and X.L. performed the experiments. Z.L. and K.P. analyzed the data. S.C. provided resources. Z.L. wrote the manuscript. Z.W., S.H. and R.L. revised the manuscript. All authors have read and agreed to the published version of the manuscript.

Funding: This work was supported by the Natural Science Foundation of Jiangsu Province (BK20180900 and BK20200316), the Priority Academic Program Development of Jiangsu Higher Education Institutions (PAPD), and the Fundamental Research Funds for the Central Universities (14380470). 
Institutional Review Board Statement: Not applicable.

Informed Consent Statement: Not applicable.

Data Availability Statement: The data presented in this study are openly available in Figshare at https:/ / doi.org/10.6084/m9.figshare.14885238.v3 (accessed on 8 July 2021).

Acknowledgments: The K. pneumoniae YZ6 included in this study was kindly given by Shangshang Qin from the School of Pharmaceutical Sciences, Zhengzhou University.

Conflicts of Interest: The authors declare no conflict of interest.

\section{References}

1. San Millan, A.; Pena-Miller, R.; Toll-Riera, M.; Halbert, Z.V.; McLean, A.R.; Cooper, B.S.; MacLean, R.C. Positive selection and compensatory adaptation interact to stabilize non-transmissible plasmids. Nat. Commun. 2014, 5, 5208. [CrossRef] [PubMed]

2. Carattoli, A. Plasmids and the spread of resistance. Int. J. Med. Microbiol. 2013, 303, 298-304. [CrossRef]

3. Baltrus, D.A. Exploring the costs of horizontal gene transfer. Trends Ecol. Evol. 2013, 28, 489-495. [CrossRef]

4. Harrison, P.W.; Lower, R.P.; Kim, N.K.; Young, J.P. Introducing the bacterial 'chromid': Not a chromosome, not a plasmid. Trends Microbiol. 2010, 18, 141-148. [CrossRef]

5. Carroll, A.C.; Wong, A. Plasmid persistence: Costs, benefits, and the plasmid paradox. Can. J. Microbiol. 2018, 64, 293-304. [CrossRef]

6. Cottell, J.L.; Webber, M.A.; Piddock, L.J. Persistence of transferable extended-spectrum-beta-lactamase resistance in the absence of antibiotic pressure. Antimicrob. Agents Chemother. 2012, 56, 4703-4706. [CrossRef] [PubMed]

7. Porse, A.; Schonning, K.; Munck, C.; Sommer, M.O. Survival and Evolution of a Large Multidrug Resistance Plasmid in New Clinical Bacterial Hosts. Mol. Biol. Evol. 2016, 33, 2860-2873. [CrossRef]

8. Kamruzzaman, M.; Wu, A.Y.; Iredell, J.R. Biological Functions of Type II Toxin-Antitoxin Systems in Bacteria. Microorganisms 2021, 9, 1276. [CrossRef] [PubMed]

9. Wu, A.Y.; Kamruzzaman, M.; Iredell, J.R. Specialised functions of two common plasmid mediated toxin-antitoxin systems, ccdAB and pemIK, in Enterobacteriaceae. PLoS ONE 2020, 15, e0230652. [CrossRef]

10. Xie, M.; Li, R.; Liu, Z.; Chan, E.W.C.; Chen, S. Recombination of plasmids in a carbapenem-resistant NDM-5-producing clinical Escherichia coli isolate. J. Antimicrob. Chemother. 2018, 73, 1230-1234. [CrossRef]

11. Liu, Z.; Xiao, X.; Liu, Y.; Li, R.; Wang, Z. Recombination of NDM-5-producing plasmids mediated by IS26 among Escherichia coli. Int. J. Antimicrob. Agents 2020, 55, 105815. [CrossRef]

12. Li, R.; Lu, X.; Peng, K.; Liu, Z.; Li, Y.; Liu, Y.; Xiao, X.; Wang, Z. Deciphering the Structural Diversity and Classification of the Mobile Tigecycline Resistance Gene tet(X)-Bearing Plasmidome among Bacteria. Msystems 2020, 5, e00134-20. [CrossRef] [PubMed]

13. Li, R.; Lu, X.; Peng, K.; Liu, Y.; Xiao, X.; Wang, Z. Reorganization of mcr-1-bearing large MDR plasmids resolved by nanopore sequencing. J. Antimicrob. Chemother. 2020, 75, 1645-1647. [CrossRef] [PubMed]

14. He, D.; Zhu, Y.; Li, R.; Pan, Y.; Liu, J.; Yuan, L.; Hu, G. Emergence of a hybrid plasmid derived from IncN1-F33:A-:B- and mcr-1-bearing plasmids mediated by IS26. J. Antimicrob. Chemother. 2019, 74, 3184-3189. [CrossRef] [PubMed]

15. Chavda, K.D.; Chen, L.; Jacobs, M.R.; Rojtman, A.D.; Bonomo, R.A.; Kreiswirth, B.N. Complete sequence of a bla(KPC)-harboring cointegrate plasmid isolated from Escherichia coli. Antimicrob. Agents Chemother. 2015, 59, 2956-2959. [CrossRef] [PubMed]

16. Liu, P.; Li, P.; Jiang, X.; Bi, D.; Xie, Y.; Tai, C.; Deng, Z.; Rajakumar, K.; Ou, H.Y. Complete genome sequence of Klebsiella pneumoniae subsp. pneumoniae HS11286, a multidrug-resistant strain isolated from human sputum. J. Bacteriol. 2012, 194, $1841-1842$. [CrossRef]

17. Li, R.; Xie, M.; Liu, L.; Huang, Y.; Wu, X.; Wang, Z.; Chan, E.W.C.; Chen, S. Characterisation of a cointegrate plasmid harbouring blaNDM-1 in a clinical Salmonella Lomita strain. Int. J. Antimicrob. Agents 2020, 55, 105817. [CrossRef]

18. Zhao, J.; Zhang, Y.; Fan, Y.; Han, J.; Xiong, Z.; Liu, X.; Li, B.; Lu, B.; Cao, B. Characterization of an NDM-5-producing hypervirulent Klebsiella pneumoniae sequence type 65 clone from a lung transplant recipient. Emerg. Microbes Infect. 2021, 10, 396-399. [CrossRef]

19. Li, X.; Mu, X.; Zhang, P.; Zhao, D.; Ji, J.; Quan, J.; Zhu, Y.; Yu, Y. Detection and characterization of a clinical Escherichia coli ST3204 strain coproducing NDM-16 and MCR-1. Infect. Drug Resist. 2018, 11, 1189-1195. [CrossRef]

20. Hunter, S.B.; Vauterin, P.; Lambert-Fair, M.A.; Van Duyne, M.S.; Kubota, K.; Graves, L.; Wrigley, D.; Barrett, T.; Ribot, E. Establishment of a universal size standard strain for use with the PulseNet standardized pulsed-field gel electrophoresis protocols: Converting the national databases to the new size standard. J. Clin. Microbiol. 2005, 43, 1045-1050. [CrossRef]

21. Bertani, G. Lysogeny at mid-twentieth century: P1, P2, and other experimental systems. J. Bacteriol. 2004, 186, 595-600. [CrossRef]

22. Ma, T.; Fu, J.; Xie, N.; Ma, S.; Lei, L.; Zhai, W.; Shen, Y.; Sun, C.; Wang, S.; Shen, Z.; et al. Fitness Cost of blaNDM-5-Carrying p3R-IncX3 Plasmids in Wild-Type NDM-Free Enterobacteriaceae. Microorganisms 2020, 8, 377. [CrossRef]

23. De Gelder, L.; Ponciano, J.M.; Joyce, P.; Top, E.M. Stability of a promiscuous plasmid in different hosts: No guarantee for a long-term relationship. Microbiology 2007, 153, 452-463. [CrossRef] 
24. Bankevich, A.; Nurk, S.; Antipov, D.; Gurevich, A.A.; Dvorkin, M.; Kulikov, A.S.; Lesin, V.M.; Nikolenko, S.I.; Pham, S.; Prjibelski, A.D.; et al. SPAdes: A new genome assembly algorithm and its applications to single-cell sequencing. J. Comput. Biol. 2012, 19, 455-477. [CrossRef]

25. Seemann, T. snippy: Fast Bacterial Variant Calling from NGS Reads. Available online: https://github.com/tseemann/snippy (accessed on 4 August 2020).

26. Kolmogorov, M.; Yuan, J.; Lin, Y.; Pevzner, P.A. Assembly of long, error-prone reads using repeat graphs. Nat. Biotechnol. 2019, 37, 540-546. [CrossRef]

27. Alikhan, N.F.; Petty, N.K.; Ben Zakour, N.L.; Beatson, S.A. BLAST Ring Image Generator (BRIG): Simple prokaryote genome comparisons. BMC Genom. 2011, 12, 402. [CrossRef]

28. Sullivan, M.J.; Petty, N.K.; Beatson, S.A. Easyfig: A genome comparison visualizer. Bioinformatics 2011, 27, 1009-1010. [CrossRef] [PubMed]

29. Ni, S.; Li, B.; Tang, K.; Yao, J.; Wood, T.K.; Wang, P.; Wang, X. Conjugative plasmid-encoded toxin-antitoxin system PrpT/PrpA directly controls plasmid copy number. Proc. Natl. Acad. Sci. USA 2021, 118, e2011577118. [CrossRef] [PubMed]

30. Partridge, S.R.; Kwong, S.M.; Firth, N.; Jensen, S.O. Mobile Genetic Elements Associated with Antimicrobial Resistance. Clin. Microbiol. Rev. 2018, 31, e00088-17. [CrossRef] [PubMed]

31. Yang, X.; Ye, L.; Chan, E.W.; Zhang, R.; Chen, S. Tracking Recombination Events That Occur in Conjugative Virulence Plasmid p15WZ-82_Vir during the Transmission Process. Msystems 2020, 5, e00140-20. [CrossRef] [PubMed]

32. Zhao, Q.Y.; Li, W.; Cai, R.M.; Lu, Y.W.; Zhang, Y.; Cai, P.; Webber, M.A.; Jiang, H.X. Mobilization of Tn1721-like structure harboring blaCTX-M-27 between P1-like bacteriophage in Salmonella and plasmids in Escherichia coli in China. Vet. Microbiol. 2021, 253, 108944. [CrossRef]

33. Tang, Y.; Li, G.; Liang, W.; Shen, P.; Zhang, Y.; Jiang, X. Translocation of Carbapenemase Gene blaKPC-2 both Internal and External to Transposons Occurs via Novel Structures of Tn1721 and Exhibits Distinct Movement Patterns. Antimicrob. Agents Chemother. 2017, 61, e01151-17. [CrossRef]

34. Li, G.; Zhang, Y.; Bi, D.; Shen, P.; Ai, F.; Liu, H.; Tian, Y.; Ma, Y.; Wang, B.; Rajakumar, K.; et al. First report of a clinical, multidrug-resistant Enterobacteriaceae isolate coharboring fosfomycin resistance gene fosA3 and carbapenemase gene blaKPC-2 on the same transposon, Tn1721. Antimicrob. Agents Chemother. 2015, 59, 338-343. [CrossRef] [PubMed]

35. Frech, G.; Schwarz, S. Plasmid-encoded tetracycline resistance in Salmonella enterica subsp. enterica serovars choleraesuis and typhimurium: Identification of complete and truncated Tn1721 elements. FEMS Microbiol. Lett. 1999, 176, 97-103. [CrossRef] [PubMed]

36. Hua, X.; Zhang, L.; Moran, R.A.; Xu, Q.; Sun, L.; van Schaik, W.; Yu, Y. Cointegration as a mechanism for the evolution of a KPC-producing multidrug resistance plasmid in Proteus mirabilis. Emerg. Microbes Infect. 2020, 9, 1206-1218. [CrossRef]

37. Lee, H.; Shin, J.; Chung, Y.J.; Park, M.; Kang, K.J.; Baek, J.Y.; Shin, D.; Chung, D.R.; Peck, K.R.; Song, J.H.; et al. Co-introduction of plasmids harbouring the carbapenemase genes, blaNDM-1 and blaOXA-232, increases fitness and virulence of bacterial host. $J$. Biomed. Sci. 2020, 27, 8. [CrossRef] [PubMed]

38. Perez-Gallego, M.; Torrens, G.; Castillo-Vera, J.; Moya, B.; Zamorano, L.; Cabot, G.; Hultenby, K.; Alberti, S.; Mellroth, P.; Henriques-Normark, B.; et al. Impact of AmpC Derepression on Fitness and Virulence: The Mechanism or the Pathway? mBio 2016, 7, e01783-16. [CrossRef]

39. Costerton, J.W.; Stewart, P.S.; Greenberg, E.P. Bacterial biofilms: A common cause of persistent infections. Science 1999, 284, 1318-1322. [CrossRef]

40. Weingarten, R.A.; Johnson, R.C.; Conlan, S.; Ramsburg, A.M.; Dekker, J.P.; Lau, A.F.; Khil, P.; Odom, R.T.; Deming, C.; Park, M.; et al. Genomic Analysis of Hospital Plumbing Reveals Diverse Reservoir of Bacterial Plasmids Conferring Carbapenem Resistance. MBio 2018, 9, e02011-17. [CrossRef]

41. Savage, V.J.; Chopra, I.; O'Neill, A.J. Staphylococcus aureus biofilms promote horizontal transfer of antibiotic resistance. Antimicrob. Agents Chemother. 2013, 57, 1968-1970. [CrossRef]

42. Wein, T.; Hulter, N.F.; Mizrahi, I.; Dagan, T. Emergence of plasmid stability under non-selective conditions maintains antibiotic resistance. Nat. Commun. 2019, 10, 2595. [CrossRef] [PubMed]

43. Bouma, J.E.; Lenski, R.E. Evolution of a bacteria/plasmid association. Nature 1988, 335, 351-352. [CrossRef]

44. Harrison, E.; Guymer, D.; Spiers, A.J.; Paterson, S.; Brockhurst, M.A. Parallel compensatory evolution stabilizes plasmids across the parasitism-mutualism continuum. Curr. Biol. 2015, 25, 2034-2039. [CrossRef]

45. Heuer, H.; Fox, R.E.; Top, E.M. Frequent conjugative transfer accelerates adaptation of a broad-host-range plasmid to an unfavorable Pseudomonas putida host. FEMS Microbiol. Ecol. 2007, 59, 738-748. [CrossRef] [PubMed]

46. Knopp, M.; Andersson, D.I. Amelioration of the Fitness Costs of Antibiotic Resistance Due To Reduced Outer Membrane Permeability by Upregulation of Alternative Porins. Mol. Biol. Evol. 2015, 32, 3252-3263. [CrossRef]

47. Bredin, J.; Saint, N.; Mallea, M.; De, E.; Molle, G.; Pages, J.M.; Simonet, V. Alteration of pore properties of Escherichia coli OmpF induced by mutation of key residues in anti-loop 3 region. Biochem. J. 2002, 363, 521-528. [CrossRef] [PubMed] 